\title{
Impact of Interstitial Lung Disease on Mortality in ANCA-associated Vasculitis: A Systematic Literature Review and Meta-analysis
}

Peining Zhou ( $\sim$ zhoupeiningmail@163.com)

Peking University First Hospital https://orcid.org/0000-0002-7203-2998

Jing Ma

Peking University First Hospital

Guangfa Wang

Peking University First Hospital

\section{Research}

Keywords: ANCA-associated vasculitis, Interstitial lung disease, mortality

Posted Date: December 3rd, 2020

DOI: https://doi.org/10.21203/rs.3.rs-117281/v1

License: () (i) This work is licensed under a Creative Commons Attribution 4.0 International License. Read Full License 


\section{Abstract}

Background: Several retrospective series and case reports have described the association of interstitial lung disease (ILD) and ANCA-associated vasculitis $\triangle A A V \bigotimes$. However, the relationship between the ILD and mortality in AAV patients have not been established so far. This study aims to estimate the relevance of AAV-associated-ILD $₫ A A V$-ILD囚and mortality risk by conducting a systematic review and meta-analysis.

Methods: A comprehensive systematic review was conducted in accordance with the guidelines of PRISMA (Preferred Reporting Items for Systematic Reviews and Meta-analyses). PubMed, Embase.com and the Cochrane Library (Wiley) were searched for original observational studies. Summary estimates were derived with a random-effects model and reported as risk ratio $\mathbb{R R} \bigotimes$, tested for publication bias and heterogeneity.

Results: Ten retrospective cohort studies were included, comprising 526 AAV-ILD patients enrolled from 1974 to 2018. Meta-analysis yielded a pooled RR of 2.90 (95\% confidence interval 1.77 to 4.74 ) for death among those with AAV-ILD compared with control group. Sensitivity analysis suggested that the meta-RR result was not skewed by a single dominant study.

Conclusion: ILD might be associated with a higher mortality risk in AAV patients.

\section{Background}

Anti-neutrophil cytoplasmic antibodies (ANCA) vasculitis are immune-mediated disease that is primarily characterized by ANCA positivity and affects various organs, such as the lungs, kidneys, skin, and nervous system. Based on clinical phenotypes and pathologies, cases of AAV are divided into three diseases: microscopic polyangiitis (MPA), granulomatosis with polyangiitis (GPA, formerly called Wegener's granulomatosis) and eosinophilic granulomatosis with polyangiitis (EGPA, formerly called Churg-Strauss syndrome). ${ }^{[1]}$

Interstitial lung disease (ILD) is a rare condition, whose prevalence and incidence rates were reported to be $97.9 / 100,000$ and 19.4/100,000, respectively, in a recent French study. ${ }^{[2]}$ Progressive fibrosing ILD generates two main computed-tomography (CT) scan patterns: usual interstitial pneumonitis (UIP) and nonspecific interstitial pneumonia (NSIP). UIP is characterized by reticular changes and honeycombing in the subpleural sections of the lower lobes on high resolution computed tomography (HRCT) whereas the radiological features of NSIP often include ground glass shadowing with features of alveolar inflammation. ${ }^{[3]}$

During the last few years, an increasing number of publications have reported the association between ILD and AAV, particularly in anti-MPO positive AAV[4-

16]. ILD occurs concurrently or antedates MPA in the majority of affected individuals. ${ }^{[4,5,7,17-24]}$. The most frequent CT scan pattern is usual interstitial pneumonia (UIP) in 43 to $83 \%$, but other patterns (e.g. suggestive of NSIP) may be seen $(5-31 \%) .{ }^{[4,5,25]}$ Progressive fibrosing ILD occurs in many systemic diseases (e.g. sarcoidosis, systemic sclerosis, rheumatoid arthritis or myositis) and is associated with shorter survival[26], Several case series have reported ILD seems to be a major prognostic factor in AAV patients. ${ }^{[8-11,13-16,27-30]}$ To further investigation, we performed a systematic literature review and metaanalysis to estimate the impact of ILD had on mortality.

\section{Methods}

A comprehensive systematic review was conducted in accordance with the guidelines of PRISMA (Preferred Reporting Items for Systematic Reviews And Meta-analyses). ${ }^{[31]}$ The review protocol was registered on 26 March 2020 in the International Prospective Register of Systematic Reviews database.

\section{Search strategies:}

The databases PubMed (N.L.M.), Embase.com and the Cochrane Library (Wiley) were searched by two authors (P.Z and J.M.) from their inception to March 2020. In brief, three search concepts were combined with the Boolean operator 'AND': (1) ANCA-associated vasculitis (AAV) or vasculitis,(2) interstitial lung disease(ILD)or pulmonary fibrosis $\square$ PFワand (3) mortality or survival rate. Search terms included indexed terms from Medical Subject Headings (MeSH) in PubMed, EMtree in EMBASE.com as well as free-text terms. The search strategy is available in Supplemental data 1. All identified studies were combined in a single reference manager file (EndNote). Abstracts for all articles of interest were reviewed for relevance, which is those that focused on the association between ILD and AAV and mortality or survival data in AAV-ILD patients. Full papers of selected abstracts were retrieved and assessed for eligibility based on the inclusion criteria.

Observational studies that met the following criteria were assessed: (1) clearly defined AAV identified by either the American College of Rheumatology 1990 classification criteria or the 2012 Chapel Hill Consensus Conference disease definitions, and (2) provided ILD-related risk ratios or hazard ratios, or can be calculated from the studies.

Criteria were used for the diagnosis of ILD mostly depending on radiological evidence on chest X-ray and HRCT (such as reticular abnormality or honeycombing with or without traction bronchiectasis), which can be combined with other examination results(such as lung function testing and/or histopathology). The control group was considered to diagnose AAV with no ILD but were likely to have other common clinical manifestations, such as sinus pain, nasal discharge, or crusting, ear pain, or deafness, foot drop or wrist drop, hematuria and oliguria. Articles analyzing connective tissue diseaseassociated ILD (CTD-ILD) or collagen vascular disease associated ILD (CVD-ILD), and not specifically AAV-ILD, were excluded.

We also searched the reference lists of identified papers and conference abstracts for additional relevant publications. In cases of duplicate data used in more than one study, the sample with the most up-to-date data was selected for review. No additional exclusions were made on the basis of study design, quality of 
the study, or duration of follow-up. The study identification and selection process are summarized in Supplemental data 2.

\section{Data extraction:}

Data were collected through a standardized extraction sheet. One reviewer (P.Z.) extracted and the second reviewer (J.M.) checked the data. Disagreements were discussed and resolved. From eligible studies, the following data were collected: year of publication, enrolment period, study design, country, sample size and demographics, mean follow-up duration, number of AAV-ILD patients, method for ILD diagnosis, survival or mortality data.

\section{Quality assessment:}

Study quality was assessed using the Newcastle-Ottawa scale (NOS). ${ }^{[32]}$ In this scale, studies are scored across three categories: selection of study groups (four questions), comparability of study groups (one question) and ascertainment of the outcome of interest (three questions) for cohort studies. Two authors (P.Z and J.M) performed quality scoring independently, with differences resolved by consensus.

\section{Statistical analyses:}

In this meta analyses, the outcomes of interest were pooled RR for AAV-ILD, HR were assumed to be numerically the same as the RR. Forest plots and pooled estimates were derived with inverse variance weighting and a random-effects model. The heterogeneity among studies was tested with Cochran's $Q$ test. Significant heterogeneity was defined as a value of $P<0.10$. The $\mathrm{I}^{2}$-statistic was used to quantify the heterogeneity. ${ }^{[33]}$ Source of heterogeneity was determined by subgroup analysis. All included studies were stratified according to enrolment periods (2010-2015 vs 2016-2020), center (single center vs multicenter), ethnicity (Asian vs non-Asian), covariates (covariates vs non-covariate),follow-up period ( $\leq 48 \mathrm{months}$ vs $₫ 48 \mathrm{months}$ ), and UIP pattern. Furthermore, a univariate meta-regression analysis was then used to interpret the difference in RRs between the subgroups. ${ }^{\text {[4] }}$ We evaluated the robustness of the results using jackknife sensitivity analysis, by repeated meta-RR analyses with removal of a single study in succession each time. ${ }^{[35]} \mathrm{A}$ funnel plot was conducted to detect publication bias (i.e. bias resulting from the greater likelihood of studies with positive results to be published compared with negative results) or the small-study effect (i.e. a tendency for treatment effect estimates in small studies to differ from those in larger studies) ${ }^{[36]}$. Furthermore, we used Egger's regression as an objective, quantitative test statistic to test for the presence of asymmetry in the data. ${ }^{[37]}$ Data analysis was performed with Review manager (RevMan) version 5.3 and Stata statistical software V.StataSE14 (stataCorp LLC, College Station, Texas, USA).

\section{Results}

A total of nine hundred records were processed for a final selection of ten articles. Most records were irrelevant, and of the 54 records were reviewed based on full text. The most frequent reasons for exclusion upon full text review were studies focusing on ANCA-positive ILD or lack of mortality data. Finally, 44 records were removed according to inclusion criteria and the remaining 10 studies were included in the meta-analysis. A list of the excluded articles is provided in Supplemental data 3 .

\section{Study characteristics:}

Characteristic features of the studies included in this meta-analysis are summarized in Table 1. The studies were published between 2010 and 2019 , enrolled from 1974 to 2018; All of these were retrospective cohort studies, four of which were from multicenter. Locations included 6 Asian studies, of which 4 from Japan, 1 from China and 1 from Korea, and the remaining studies came from Greece, London, Germany, Argentina respectively. The sample sizes in the individual studies ranged from 9 to 301 AAV-ILD patients and 526 in total. One study used three other control groups, including alveolar hemorrhage, bronchial asthma and pulmonary granuloma. The other study used bronchiectasis as an additional control group. Five studies adjusted mortality for BVAS, age, cardiovascular involvement, alveolar hemorrhage, serum creatinine level et al. The included studies were of high quality, scoring 7 to 9 points according to the Newcastle-Ottawa Scale. 
Table 1

Characteristic features of the included studies

\begin{tabular}{|c|c|c|c|c|c|c|c|c|c|c|c|c|}
\hline Author & Year & Country & $\begin{array}{l}\text { Research } \\
\text { Center }\end{array}$ & $\begin{array}{l}\text { Cohort } \\
\text { Type }\end{array}$ & Period & $\begin{array}{l}\text { Follow- } \\
\text { up(months) }\end{array}$ & $\begin{array}{l}\text { Number } \\
\text { of } \\
\text { Patients }\end{array}$ & $\begin{array}{l}\text { Number } \\
\text { of ILD } \\
\text { Patients }\end{array}$ & $\begin{array}{l}\text { Number } \\
\text { of UIP } \\
\text { Patients }\end{array}$ & $\begin{array}{l}\text { Number } \\
\text { of } \\
\text { Deaths } \\
\text { (ILD vs } \\
\text { control) }\end{array}$ & $\begin{array}{l}\text { Survival } \\
\text { rate of } \\
\text { control } \\
\text { group }\end{array}$ & $\begin{array}{l}\text { Survival } \\
\text { rate of IL }\end{array}$ \\
\hline Tzelepis & 2010 & Greece & $M$ & $\mathrm{~N}-\mathrm{I}$ & $\begin{array}{l}2003- \\
2008\end{array}$ & 38.0 & MPA 33 & 13 & 7 & 6 vs 1 & $\mathrm{~N} / \mathrm{A}$ & $\mathrm{N} / \mathrm{A}$ \\
\hline \multirow[t]{2}{*}{ Arulkumaran } & 2011 & London & $S$ & $\mathrm{~N}-\mathrm{I}$ & $\begin{array}{l}1974- \\
2009\end{array}$ & 61.7 & $\begin{array}{l}\text { MPA } \\
194\end{array}$ & 14 & 8 & $\begin{array}{l}10 \text { vs } \\
70\end{array}$ & 1-year: 85\%; & 1-year:5( \\
\hline & & & & & & & & & & & $\begin{array}{l}5- \\
\text { years:61\%. }\end{array}$ & 5-years: 2 \\
\hline Ahn & 2012 & Korea & $S$ & I & $\begin{array}{l}1994- \\
2009\end{array}$ & 46.1 & MPA 55 & 13 & 11 & 4 vs 2 & N/A & $\mathrm{N} / \mathrm{A}$ \\
\hline \multirow[t]{2}{*}{ Fernandez } & 2015 & Argentina & $S$ & $\mathrm{~N} / \mathrm{A}$ & $\begin{array}{l}1998- \\
2013\end{array}$ & 61.2 & MPA 28 & 9 & 8 & 4 vs 0 & $\begin{array}{l}\text { 1-year: } \\
100 \%\end{array}$ & $\begin{array}{l}1- \\
\text { year:92. }\end{array}$ \\
\hline & & & & & & & & & & & $\begin{array}{l}\text { 5-years: } \\
101 \%\end{array}$ & $\begin{array}{l}5- \\
\text { years:92 }\end{array}$ \\
\hline \multirow[t]{2}{*}{ Hirayama } & 2015 & Japan & M & N/A & $\begin{array}{l}1989- \\
2007\end{array}$ & $\mathrm{~N} / \mathrm{A}$ & $\begin{array}{l}\text { AAV } \\
1147\end{array}$ & 301 & $\mathrm{~N} / \mathrm{A}$ & $\begin{array}{l}118 \text { vs } \\
272\end{array}$ & $\begin{array}{l}1- \\
\text { year:73.5\%; }\end{array}$ & $\begin{array}{l}\text { 1-year: } \\
69.9 \%\end{array}$ \\
\hline & & & & & & & & & & & $\begin{array}{l}5- \\
\text { years:60.2\% }\end{array}$ & $\begin{array}{l}5- \\
\text { years:50 }\end{array}$ \\
\hline Ono & 2015 & Japan & M & N/A & $\begin{array}{l}2001- \\
2013\end{array}$ & 38.6 & AAV 79 & 14 & 14 & $\mathrm{~N} / \mathrm{A}$ & N/A & $\mathrm{N} / \mathrm{A}$ \\
\hline Schirmer & 2016 & Germany & $S$ & $\mathrm{~N}-\mathrm{I}$ & $\begin{array}{l}1999- \\
2013\end{array}$ & 72.0 & $\begin{array}{l}\text { MPA } \\
144\end{array}$ & 17 & $\mathrm{~N} / \mathrm{A}$ & $\mathrm{N} / \mathrm{A}$ & N/A & $\mathrm{N} / \mathrm{A}$ \\
\hline \multirow[t]{2}{*}{ Tashiro } & 2017 & Japan & $S$ & N/A & $\begin{array}{l}2004- \\
2011\end{array}$ & 52.9 & MPA 45 & 23 & $\mathrm{~N} / \mathrm{A}$ & 5 vs 4 & $\begin{array}{l}1- \\
\text { year:90.9\% }\end{array}$ & $\begin{array}{l}1- \\
\text { year:78. }\end{array}$ \\
\hline & & & & & & & & & & & $\begin{array}{l}5- \\
\text { years:81.9\% }\end{array}$ & $\begin{array}{l}5- \\
\text { years:78 }\end{array}$ \\
\hline Ono & 2019 & Japan & M & I & $\begin{array}{l}2012- \\
2018\end{array}$ & 24.2 & $\begin{array}{l}\text { AAV } \\
143\end{array}$ & 63 & $\mathrm{~N} / \mathrm{A}$ & $\mathrm{N} / \mathrm{A}$ & N/A & $\mathrm{N} / \mathrm{A}$ \\
\hline Shi & 2019 & China & $\mathrm{S}$ & I & $\begin{array}{l}2012- \\
2017\end{array}$ & 17.0 & $\begin{array}{l}\text { MPA } \\
124\end{array}$ & 59 & $\mathrm{~N} / \mathrm{A}$ & $\begin{array}{l}20 \text { vs } \\
26\end{array}$ & $\begin{array}{l}1- \\
\text { year:89.2\% }\end{array}$ & $\begin{array}{l}1- \\
\text { year:66. }\end{array}$ \\
\hline
\end{tabular}

N/A: not available; ILD: interstitial lung disease; UIP: usual interstitial pneumonia; AAV: ANCA-associated vasculitis; MPA: microscopic polyangiitis; I: inceptior non-inception cohort, S: single center, M: multicenter

\section{Causes of death:}

Table 2 details the causes of death in the included studies if were available. The majority of the deaths were respiratory failure and infectious pneumonia.

Table 2

Causes of death

\begin{tabular}{|c|c|c|c|}
\hline Author & $\begin{array}{l}\text { No. of } \\
\text { deaths(n) }\end{array}$ & $\begin{array}{l}\text { AA-ILD } \\
\text { cohort(n) }\end{array}$ & Causes of death(n) \\
\hline $\begin{array}{l}\text { Tzelepis, G. E et } \\
\text { al. }\end{array}$ & 6 & 13 & 4 respiratory failure, 1 lung cancer, 1 sepsis. \\
\hline $\begin{array}{l}\text { Arulkumaran, } \mathrm{N} \\
\text { et al. }\end{array}$ & 10 & 14 & 5 respiratory failure, 3 multi-organs failure/generalized sepsis, 2 unknown \\
\hline $\begin{array}{l}\text { Fernandez } \\
\text { Casares, M } \\
\text { et al. }\end{array}$ & 4 & 9 & 4 respiratory failure \\
\hline $\begin{array}{l}\text { Hirayama, } \mathrm{K} \text { et } \\
\text { al. }\end{array}$ & 118 & 301 & $\begin{array}{l}21 \text { pulmonary involvements due to vasculitis; } 42 \text { both pulmonary involvements and infectious diseases; } \\
39 \text { infectious diseases; } 16 \text { others. }\end{array}$ \\
\hline Tashiro, $\mathrm{H}$ et al. & 5 & 23 & 1 ARDS, 1 exacerbation of $\mathrm{DAH}, 1$ multiple organ failure, 1 unknown sudden death, 1 exacerbation of IP \\
\hline Total & 143 & 360 & $\mathrm{~N} / \mathrm{A}$ \\
\hline
\end{tabular}

Impact of ILD on mortality in AAV: 
We pooled the results of all ten studies comparing mortality risk between AAV-ILD and control groups and observed an increase in mortality among ILD, [risk ratio $(R R)=2.90 ; 95 \%$ confidence interval $(C l) 1.77-4.74]$. There was a significant heterogeneity among the studies, $I^{2}=72 \%(p<0.0001) .(F i g .1)$

\section{Sensitivity analysis:}

The results of jackknife sensitivity analysis are shown in Table 3. The RRs remained significantly increased regardless of each study exclusion sequentially, with the value estimated ranging from 2.55 to 3.60 in all analyses. This suggested that the meta-RR result was robust and not skewed by a single dominant study.

Table 3

Sensitivity analysis

\begin{tabular}{|llll|}
\hline Authors & Year & RR $(95 \%$ Cl $)$ & Study excluded, meta-RR $(95 \%$ Cl) \\
\hline Tzelepis, G. E et al. & 2010 & $9.23[1.25,68.11]$ & $2.70[1.65,4.42]$ \\
\hline Arulkumaran, N et al. & 2011 & $1.84[1.26,2.68]$ & $3.60[1.81,7.15]$ \\
\hline Ahn, J. K.et al. & 2012 & $5.33[1.12,25.44]$ & $2.75[1.66,4.56]$ \\
\hline Fernandez Casares, M et al. & 2015 & $18.00[1.07,302.41]$ & $2.72[1.67,4.43]$ \\
\hline Hirayama, K et al. & 2015 & $1.22[1.03,1.45]$ & $3.47[2.08,5.78]$ \\
\hline Ono, N et al. & 2015 & $7.85[2.14,28.79]$ & $2.55[1.57,4.12]$ \\
\hline Schirmer, J. H et al.. & 2016 & $4.04[1.21,13.49]$ & $2.79[1.67,4.67]$ \\
\hline Tashiro, H et al. & 2017 & $1.20[0.37,3.88]$ & $3.24[1.90,5.53]$ \\
\hline Shi, J et al. & 2019 & $3.15[1.44,6.90]$ & $2.87[1.68,4.88]$ \\
\hline Ono, N et al & 2019 & $7.55[1.70,33.47]$ & $2.63[1.61,4.30]$ \\
\hline all studies & $/$ & $2.90[1.77,4.74]$ & $/$ \\
\hline
\end{tabular}

\section{Subgroup analysis:}

Subgroup analyses showed that meta-RR were higher in non-Asian group, multicenter group, adjusted covariates group, less follow-up period group, UIP group and in cohorts enrolled after 2015. However, meta regression analysis showed that there was no significant difference in subgroups except the follow-up period group. [Figure.2, Table 4]

Table 4

Subgroup analysis and meta-regression analysis

\begin{tabular}{|llllll|}
\hline Study subset & No.cohorts & RR(95\%Cl) & I'(\%) & P(a) & P(b) \\
\hline Enrollment period & 10 & & & & 0.80 \\
\hline $2010-2015$ & 6 & $2.73[1.47,5.07]$ & 76 & 0.001 & \\
\hline $2016-2019$ & 4 & $3.06[1.60,5.84]$ & 26 & 0.25 & \\
\hline Ethnicity & 10 & & & & 0.74 \\
\hline Asian & 6 & $2.90[1.36,6.18]$ & 76 & 0.0008 & \\
\hline non-Asian & 4 & $3.53[1.43,8.74]$ & 49 & 0.11 & \\
\hline Center & 10 & & & & 0.44 \\
\hline single center & 6 & $2.49[1.56,3.97]$ & 29 & 0.22 & \\
\hline multicenter & 4 & $4.37[1.14,16.80]$ & 82 & 0.0007 & \\
\hline Covariates & 10 & & & & 0.51 \\
\hline Covariates & 6 & $3.59[1.60,8.05]$ & 79 & 0.0002 & \\
\hline no-Covariates & 4 & $2.43[1.04,5.67]$ & 45 & 0.14 & \\
\hline Follow-up period & 9 & & & & 0.04 \\
\hline$\leq 48$ months & 5 & $4.83[2.79,8.35]$ & 0 & 0.65 & \\
\hline$\square 48$ months & 4 & $2.19[1.16,4.11]$ & 34 & 0.21 & \\
\hline Total & 10 & $2.90[1.77,4.74]$ & 72 & 0.0003 & \\
\hline P(a):p value of subgroup; $\mathrm{P}(\mathrm{b}): \mathrm{p}$ value between two subgroups. & & \\
\hline
\end{tabular}


The funnel plot is shown in Fig. 3. Visual inspection of the funnel plot was asymmetrically located. Using the Egger's test for publication bias with the 10 studies, the p-value less than 0.10 indicating that there is an evidence for publication bias. We further evaluated the number of missing studies in this metaanalysis by the application of the trim and fill method and recalculated the pooled risk estimate with the addition of those missing study. There was no significant change in the value of summary statistic, suggesting that publication bias had no significant impact on the result.

\section{Discussion}

This is the first meta-analysis of observational studies assessing the mortality risk in AAV-ILD. We found a 2.9-fold increased risk of death in patients with AAV-ILD when compared with control group. The RR remained significantly increased by sensitivity analysis, suggesting that the meta-RR result was robust and not skewed by a single dominant study. Given the heterogeneity observed in the pooled risk estimate, subgroup analysis was conducted. We further compared the RRs between the two groups by meta regression analysis, which showed that the only one difference is found in the follow-up period group. However, we still hope to explore the factors that may latently affect the prognosis of AAV-ILD patients.

Of interest, mortality risks were even higher in later cohorts, that is, those with their enrolment periods that were after 2015 relative to those before 2015 , although the overall comparison between the cohorts was non-significant. Over the last few decades, as treatment of AAV has evolved, disease-related mortality rates have declined. Yet, this may not be the case in individuals with AAV-ILD. In a study by Alba et al, which revealed the mortality rates of AAV-ILD patients from 2004 to 2015, suggesting no significant improvement over time. ${ }^{[15]}$ We hypothesized that this observation may due to the lack of universally accepted treatment guidelines. The effect of treatment in ILD is still controversial right now. Some studies have showed clinical or radiological improvement in $80 \%$ of patients while on immunosuppressive therapy. ${ }^{[39,40]}$ In addition, immunosuppressive therapy might be associated with a reduction in the development of full-blown MPA, which initially presented as isolated pulmonary fibrosis with positive MPO-ANCA. ${ }^{[40,41]}$ In sharp contrast, most of the retrospective publications reported that the disease remained unchanged or deteriorated in the majority of patients, even in presence of clinically quiescent systemic vasculitis. ${ }^{[5,19,42]}$ Although no clear conclusions can be drawn about the initial treatment, it is recommended that general measures are instituted as required: supplemental oxygen, pulmonary rehabilitation and influenza and pneumococcal vaccination. In the future, novel anti-fibrotic therapies (pirfenidone and nintedanib) designed for idiopathic pulmonary fibrosis might be tested in AAV-ILD. Nintedanib, an oral tyrosine kinase inhibitor has been associated stabilization of lung fibrosis. A recent double-blind, placebo-controlled, phase 3 trial, NCT0299917, has proved the efficacy of nintedanib not only in idiopathic pulmonary fibrosis but in a broad range of fibrosing lung diseases as well. ${ }^{[43]}$ Meanwhile, clinical trials (NCT03385668 and NCT02999178) are ongoing to evaluate the effect of antifibrotic drugs(pirfenidone and nintedanib) authorized for AAV in patients with lung fibrosis. ${ }^{[16]}$

Race may also be one of the factors latent associated with AAV-ILD. Frequency of ILD in ANCA-associated vasculitis seems to be higher in Asian than in Western populations. ${ }^{[4,5,7,8]}$ In a Japanese nationwide, prospective, inception cohort study of AAV (Remission Induction Therapy in Japanese Patients with ANCA-associated Vasculitis; RemIT-JAV), 39.1\% patients had ILD. ${ }^{[44]}$ In contrast, ILD was observed in only $2.7 \%$ AAV patients at a renal vasculitis clinic in London. ${ }^{[5]}$ This discrepancy between Japan and other countries has been usually explained by the ethnicity and the predominance of MPO-ANCA. Hirayama K et al. reported that the 5 -year survival rate was $50.2 \%$ in the patients with ILD and $73.3 \%$ in those without pulmonary involvement; ILD was added as one of the predictors of 5-year mortality. In contrast, in a retrospective study by the renal vasculitis clinic in London, there was no difference in mortality between patients with AAV-ILD and those without over the same time period. ${ }^{[5]}$ On the contrary, some European studies supported that AAV-ILD patients were more likely to have a much worse prognosis. ${ }^{[4,7,10,27]}$ Therefore, we postulate that this controversy might due to small cohort size in non-Asian studies, which partly came from single center.

It was also interesting to note the trend for increased risk of death in studies that were adjusted covariates in multivariate model, RR 3.59(95\% $\mathrm{Cl} 1.60-8.05)$. One might explain that the impact of ILD was latently masked by other factors involved and relevant RR was underestimated. Several factors may confound mortality in AAV-ILD patients, mostly including age, alveolar hemorrhage, Birmingham vasculitis activity score (BVAS), and renal involvement. ${ }^{[7-10,13,14]}$ First, repeated episodes of alveolar hemorrhage could be the pathogenesis of pulmonary fibrosis. ${ }^{[19]}$ However, this may lead researchers to mistakenly attribute ILDrelated death to alveolar hemorrhage. Secondly, renal involvement was significantly more common in MPA, affecting $93 \%$ compared to $66 \%$ of those with GPA. Compared to GPA, serum creatine levels were significantly higher in those with MPA. ${ }^{[9]}$ Numerous patients eventually died from dialysis-related complications, thereby ignoring the potential impact of ILD. Moreover, pulmonary fibrosis(PF)occurs concurrently or antedates MPA in the majority of affected individuals. ${ }^{[15]}$ In previous reports, ILD precedes other vasculitis syndrome in $14-85 \%$ of patients. $[4,5,17-20,22,23,25,39,45,46]$ In those cases when pulmonary fibrosis was diagnosed before MPA, the time range from a few months to 12 years. Among the studies with short-term follow-up, the impact of ILD may be ignored for these patients has not met the diagnostic criteria of AAV yet. It is necessary for further research to explore the role of ILD in the course of AAV development by prolonging follow-up time, emphasizing on regularly radiographic images and pulmonary function tests.

Current studies suggest that ILD, especially UIP, mainly affects the long-term course. Our study confirmed that the RR-value tend to be higher in UIP group, even higher in the group which has shorter follow-up period, suggesting that ILD is not only associated with long-term prognosis, but also a risk factor for poor prognosis within 48 months. Pulmonary fibrosis frequently exists before systemic involvement, which might aggravate the possibility of respiratory failure and pulmonary infection, most of which need to use intravenous corticosteroids and immunosuppressive therapy. Among the causes of death in AAV-ILD patients, respiratory failure and infectious diseases of the ILD are among the most frequent causes of death. ${ }^{[4,5,7,12]}$ In the series of MPA patients with pulmonary

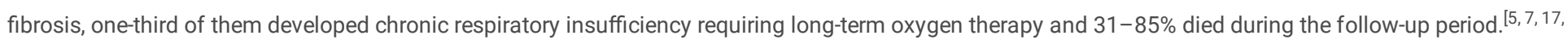
$25,41,47$. Meanwhile, Infectious complications are associated with mortality in AAV patients over the first year after diagnosis, and maintenance therapy with corticosteroids increases the incidence rate of infections. ${ }^{[8,13,28,47,48]}$ 
The strengths of our meta-analysis include the thorough systematic literature review, duplicate abstraction, careful exclusion of studies on overlapping populations, evaluation of study quality using the Newcastle-Ottawa scale. Both sensitivity and subgroup analysis were applied to evaluate the stability and heterogenous of findings. Although there is an evidence for publication bias, no significant change in the recalculated value of the summary statistic by the application of the trim and fill method, suggesting that publication bias had no significant influence on the result.

Our meta-analysis has few limitations. The follow-up period of studies is different, which may affect the result of meta-analysis. However, we have already adopted appropriate statistical methods to minimize the bias. Firstly, sensitivity analysis suggested that this conclusion is relatively stable and not affected by a single test. Secondly, we conducted subgroup analysis according to follow-up months, which reveals that each groups of RR values, regarding to follow-up period no more than 48 months and longer than 48 months, were higher than control group. Therefore, we still have reason to believe that this result has a certain reference value. Another limitation is that the values we used to assess prognostic factors were not identical, including risk ratios or hazard ratios. However, we choose the covariate adjusted HR to reduce the heterogeneity of the result as far as possible. At the same time, a particularity of this systemic review needs to be considered, for the outcome is rare in all populations and subgroups under review, one can generally ignore the distinctions among various measures of relative risks, such as odd ratios, risk ratio and hazard ratio. ${ }^{[48]}$ Moreover, no studies adjusted for respiratory physiology. Homma S et al, reported that causes of death in AAV-ILD include progressive deterioration of pulmonary function, ${ }^{[47]}$ suggesting latter studies should concentrate on the relationship between pulmonary function and mortality.

\section{Conclusion}

In summary, our meta-analysis indicated that ILD is a complication of AAV that might associated with poor prognosis. The risk of death in AAV-ILD patients remains relatively high over time, which warrants further investigation to create optimum treatment protocols for this special subpopulation of AAV patients.

\section{Abbreviations}

AAV: ANCA-associated vasculitis; ILD: interstitial lung disease; PF: pulmonary fibrosis; RR: risk ratio; MPA: microscopic polyangiitis; GPA: granulomatosis with polyangiitis; EGPA: eosinophilic granulomatosis with polyangiitis; UIP: usual interstitial pneumonia; NSIP non-specific interstitial pneumonia; HRCT: high resolution computed tomography; CTD: connective tissue disease; CVD: collagen vascular disease.

\section{Declarations \\ Contributors:}

GW planned the study. JM helped guide the study and assisted in data collection.

\section{Funding:}

No specific funding was received from any bodies in the public, commercial or not-for-profit sectors to carry out the work described in this manuscript.

\section{Competing interests:}

There are no competing interests for any author.

\section{Acknowledgements:}

We would like to thank Professor Xueying Li in the Department of Medical Statistics of Peking University First Hospital for her helpful instruction in data analysis and statistics to the research.

\section{Ethics approval and consent to participate:}

All analyses were based on previous published studies, thus no ethical approval and patient consent are required.

\section{Consent for publication:}

The manuscript has been read and approved for publication by all the authors.

\section{Availability of data and material:}

All data and materials in this manuscript are available.

\section{References}


1. C. Jennette, R. J. Falk, P. A. Bacon, et al. 2012 revised International Chapel Hill Consensus Conference Nomenclature of Vasculitides. Arthritis and rheumatism, 2013, 1:1529-0131

2. B. Duchemann, I. Annesi-Maesano, C. Jacobe de Naurois, et al. Prevalence and incidence of interstitial lung diseases in a multi-ethnic county of Greater Paris. The European respiratory journal, 2017, 2:1399-3003

3. A. Kekevian, M. E. Gershwin and C. Chang. Diagnosis and classification of idiopathic pulmonary fibrosis. Autoimmunity reviews, 2014, 4-5:508-512.18730183

4. G. E. Tzelepis, M. Kokosi, A. Tzioufas, et al. Prevalence and outcome of pulmonary fibrosis in microscopic polyangiitis. Eur Respir J, 2010, 1:116-21.09031936

5. N. Arulkumaran, N. Periselneris, G. Gaskin, et al. Interstitial lung disease and ANCA-associated vasculitis: a retrospective observational cohort study. Rheumatology (Oxford), 2011, 11:2035-43.1462-0324

6. J. K. Ahn, J. W. Hwang, J. Lee, et al. Clinical features and outcome of microscopic polyangiitis under a new consensus algorithm of ANCA-associated vasculitides in Korea. Rheumatol Int, 2012, 10:2979-86.0172-8172

7. M. Fernandez Casares, A. Gonzalez, M. Fielli, et al. Microscopic polyangiitis associated with pulmonary fibrosis. Clin Rheumatol, 2015, 7:1273-7.07703198

8. K. Hirayama, M. Kobayashi, J. Usui, et al. Pulmonary involvements of anti-neutrophil cytoplasmic autoantibody-associated renal vasculitis in Japan. Nephrol Dial Transplant, 2015, i83-93.0931-0509

9. N. Ono, H. Niiro, A. Ueda, et al. Characteristics of MPO-ANCA-positive granulomatosis with polyangiitis: a retrospective multi-center study in Japan. Rheumatol Int, 2015, 3:555-9.0172-8172

10. J. H. Schirmer, M. N. Wright, R. Vonthein, et al. Clinical presentation and long-term outcome of 144 patients with microscopic polyangiitis in a monocentric German cohort. Rheumatology (Oxford), 2016, 1:71-9.1462-0324

11. M. Yamagata, K. Ikeda, K. Tsushima, et al. Prevalence and Responsiveness to Treatment of Lung Abnormalities on Chest Computed Tomography in Patients With Microscopic Polyangiitis: A Multicenter, Longitudinal, Retrospective Study of One Hundred Fifty Consecutive Hospital-Based Japanese Patients. Arthritis Rheumatol, 2016, 3:713-23.2326-5191

12. H. Tashiro, K. Takahashi, M. Tanaka, et al. Characteristics and prognosis of microscopic polyangiitis with bronchiectasis. J Thorac Dis, 2017, 2:303309.2072-1439 (Print) 2072-1439

13. N. Ono, Y. Inoue, T. Miyamura, et al. The association of airway comorbidities with the clinical phenotypes and outcomes of ANCA-associated vasculitis patients. J Rheumatol, 2019, 0315-162X (Print)0315-162x

14. J. Shi, Q. Shen, X. M. Chen, et al. Clinical characteristics and outcomes in microscopic polyangiitis patients with renal involvement: a study of 124 Chinese patients. BMC Nephrol, 2019, 1:339.1471-2369

15. M. A. Alba, L. F. Flores-Suarez, A. G. Henderson, et al. Interstital lung disease in ANCA vasculitis. Autoimmun Rev, 2017, 7:722-729.1568-9972

16. R. Borie and B. Crestani. Antineutrophil Cytoplasmic Antibody-Associated Lung Fibrosis. Semin Respir Crit Care Med, 2018, 4:465-470.1069-3424

17. G. Foulon, P. Delaval, D. Valeyre, et al. ANCA-associated lung fibrosis: analysis of 17 patients. Respiratory medicine, 2008, 10:1392-1398.1532-3064

18. A. K. Nada, V. E. Torres, J. H. Ryu, et al. Pulmonary fibrosis as an unusual clinical manifestation of a pulmonary-renal vasculitis in elderly patients. Mayo Clinic proceedings, 1990, 6:847-856.0025-6196

19. B. Hervier, C. Pagnoux, C. Agard, et al. Pulmonary fibrosis associated with ANCA-positive vasculitides. Retrospective study of 12 cases and review of the literature. Annals of the rheumatic diseases, 2009, 3:404-407.1468-2060

20. T. Nozu, M. Kondo, K. Suzuki, et al. A comparison of the clinical features of ANCA-positive and ANCA-negative idiopathic pulmonary fibrosis patients. Respiration; international review of thoracic diseases, 2009, 4:407-415.1423-0356

21. G. M. Eschun, S. N. Mink and S. Sharma. Pulmonary interstitial fibrosis as a presenting manifestation in perinuclear antineutrophilic cytoplasmic antibody microscopic polyangiitis. Chest, 2003, 1:297-301.0012-3692

22. D. Eleftheriou, S. Katsenos, S. Zorbas, et al. Pulmonary fibrosis presenting as an early manifestation of microscopic polyangiitis. Monaldi archives for chest disease = Archivio Monaldi per le malattie del torace, 2012, 3-4:141-144.1122-0643

23. P. B. Gaudin, F. B. Askin, R. J. Falk, et al. The pathologic spectrum of pulmonary lesions in patients with anti-neutrophil cytoplasmic autoantibodies specific for anti-proteinase 3 and anti-myeloperoxidase. American journal of clinical pathology, 1995, 1:0002-9173

24. N. Kagiyama, N. Takayanagi, T. Kanauchi, et al. Antineutrophil cytoplasmic antibody-positive conversion and microscopic polyangiitis development in patients with idiopathic pulmonary fibrosis. BMJ open respiratory research, 2015, 1:e000058.2052-4439

25. H. Huang, Y. X. Wang, C. G. Jiang, et al. A retrospective study of microscopic polyangiitis patients presenting with pulmonary fibrosis in China. BMC Pulm Med, 2014, 8.1471-2466

26. W. D. Travis, U. Costabel, D. M. Hansell, et al. An official American Thoracic Society/European Respiratory Society statement: Update of the international multidisciplinary classification of the idiopathic interstitial pneumonias. American journal of respiratory and critical care medicine, 2013, 6:733-748.15354970

27. T. Maillet, T. Goletto, G. Beltramo, et al. Usual interstitial pneumonia in ANCA-associated vasculitis: A poor prognostic factor. J Autoimmun, 2020, 102338.0896-8411

28. W. Zhao, H. Dai, Y. Liu, et al. Clinical features and prognosis of microscopic polyangiitis with usual interstitial pneumonia compared with idiopathic pulmonary fibrosis. Clin Respir J, 2019, 7:460-466.1752-6981 
29. A. Manfredi, M. Sebastiani, S. Cerri, et al. Acute exacerbation of interstitial lung diseases secondary to systemic rheumatic diseases: a prospective study and review of the literature. J Thorac Dis, 2019, 4:1621-1628.2072-1439 (Print) 2072-1439

30. T. Shoda, T. Takeuchi, K. Isoda, et al. Prognostic factors for interstitial lung disease with microscopic polyangiitis. Annals of the Rheumatic Diseases, $2018,1128.1468-2060$

31. A. Liberati, D. G. Altman, J. Tetzlaff, et al. The PRISMA statement for reporting systematic reviews and meta-analyses of studies that evaluate health care interventions: explanation and elaboration. PLoS medicine, 2009, 7:e1000100.1549-1676

32. Wells GA, Shea B, O'Connell D, Peterson J, Welch V, Losos M, Tugwell P. The Newcastle-Ottawa Scale (NOS) for assessing the quality of nonrandomised studies in meta-analyses 2012.

33. Higgins JP, Thompson SG, Deeks JJ, et al. Measuring inconsistency in meta-analyses. BMJ 2003;327:557-60.

34. Thompson SG, Higgins JP. How should meta-regression analyses be undertaken and interpreted? Stat Med 2002;21:1559-73.

35. R. G. MILLER. The jackknife-a review. Biometrika, 1974, 1:1-15.0006-3444

36. C. B. Begg and J. A. Berlin. Publication bias and dissemination of clinical research. J Natl Cancer Inst, 1989, 2:107-15.0027-8874 (Print) 0027-8874

37. M. Egger, G. Davey Smith, M. Schneider, et al. Bias in meta-analysis detected by a simple, graphical test. Bmj, 1997, 7109:629-34.0959-8138 (Print) 09598138

38. G. Raghu, H. R. Collard, J. J. Egan, et al. An official ATS/ERS/JRS/ALAT statement: idiopathic pulmonary fibrosis: evidence-based guidelines for diagnosis and management. Am J Respir Crit Care Med, 2011, 6:788-824.1073-449X (Print)1073-449x

39. T. Nozu, M. Kondo, K. Suzuki, et al. A comparison of the clinical features of ANCA-positive and ANCA-negative idiopathic pulmonary fibrosis patients. Respiration, 2009, 4:407-15.0025-7931

40. C. Hosoda, T. Baba, E. Hagiwara, et al. Clinical features of usual interstitial pneumonia with anti-neutrophil cytoplasmic antibody in comparison with idiopathic pulmonary fibrosis. Respirology, 2016, 5:920-6.1323-7799

41. M. Ando, E. Miyazaki, T. Ishii, et al. Incidence of myeloperoxidase anti-neutrophil cytoplasmic antibody positivity and microscopic polyangitis in the course of idiopathic pulmonary fibrosis. Respiratory medicine, 2013, 4:608-615.1532-3064

42. S. Homma, A. Suzuki and K. Sato. Pulmonary involvement in ANCA-associated vasculitis from the view of the pulmonologist. Clin Exp Nephrol, 2013, 5:667-671.1342-1751

43. K. R. Flaherty, A. U. Wells, V. Cottin, et al. Nintedanib in Progressive Fibrosing Interstitial Lung Diseases. New England Journal of Medicine, 2019, 18:17181727.

44. K.-e. Sada, M. Yamamura, M. Harigai, et al. Classification and characteristics of Japanese patients with antineutrophil cytoplasmic antibody-associated vasculitis in a nationwide, prospective, inception cohort study. Arthritis research \& therapy, 2014, 2:R101.1478-6362

45. L. F. Flores-Suárez, N. Ruiz, L. M. Saldarriaga Rivera, et al. Reduced survival in microscopic polyangiitis patients with pulmonary fibrosis in a respiratory referral centre. Clinical rheumatology, 2015, 9:1653-1654.1434-9949

46. L. F. Flores-Suárez. Limited pulmonary MPA, a new MPA entity? A rheumatologist's perspective. Clinical and Experimental Nephrology, 2013, 5:672675.1342-17511437-7799

47. S. Homma, H. Matsushita and K. Nakata. Pulmonary fibrosis in myeloperoxidase antineutrophil cytoplasmic antibody-associated vasculitides. Respirology, 2004, 2:190-6.1323-7799 (Print) 1323-7799

48. Y.-X. Chen, H.-J. Yu, W. Zhang, et al. Analyzing fatal cases of Chinese patients with primary antineutrophil cytoplasmic antibodies-associated renal vasculitis: a 10-year retrospective study. Kidney \& blood pressure research, 2008, 5:343-349.1423-0143

49. Greenland. S Quantitative methods in the review of epidemiologic literature. Epidemiol Rev 1987;9:1-30

\section{Figures}

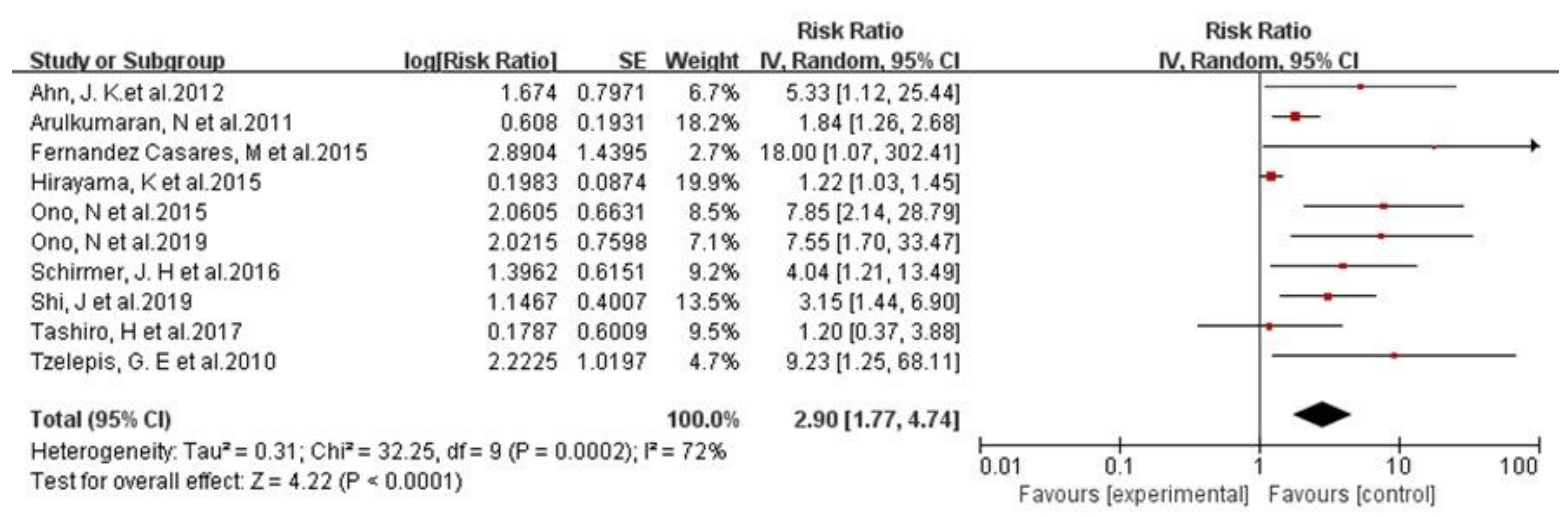

\section{Figure 1}

Meta-analysis of studies examining association between AAV-associated-ILD and mortality. 


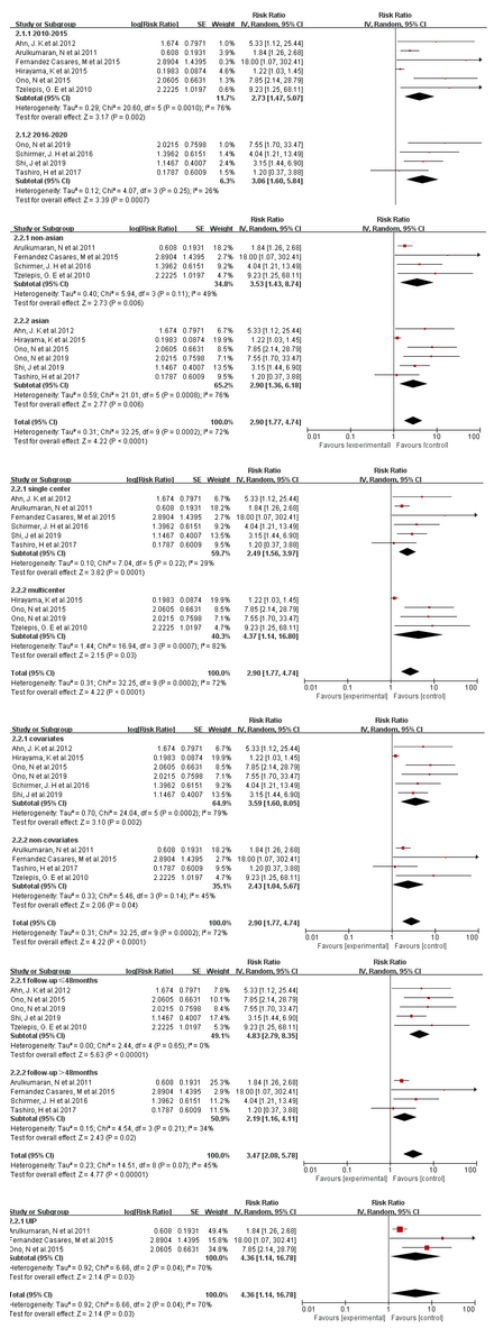

\section{Figure 2}

Meta-analysis of studies examining association between AAV-associated-ILD and mortality via subgroup analysis.

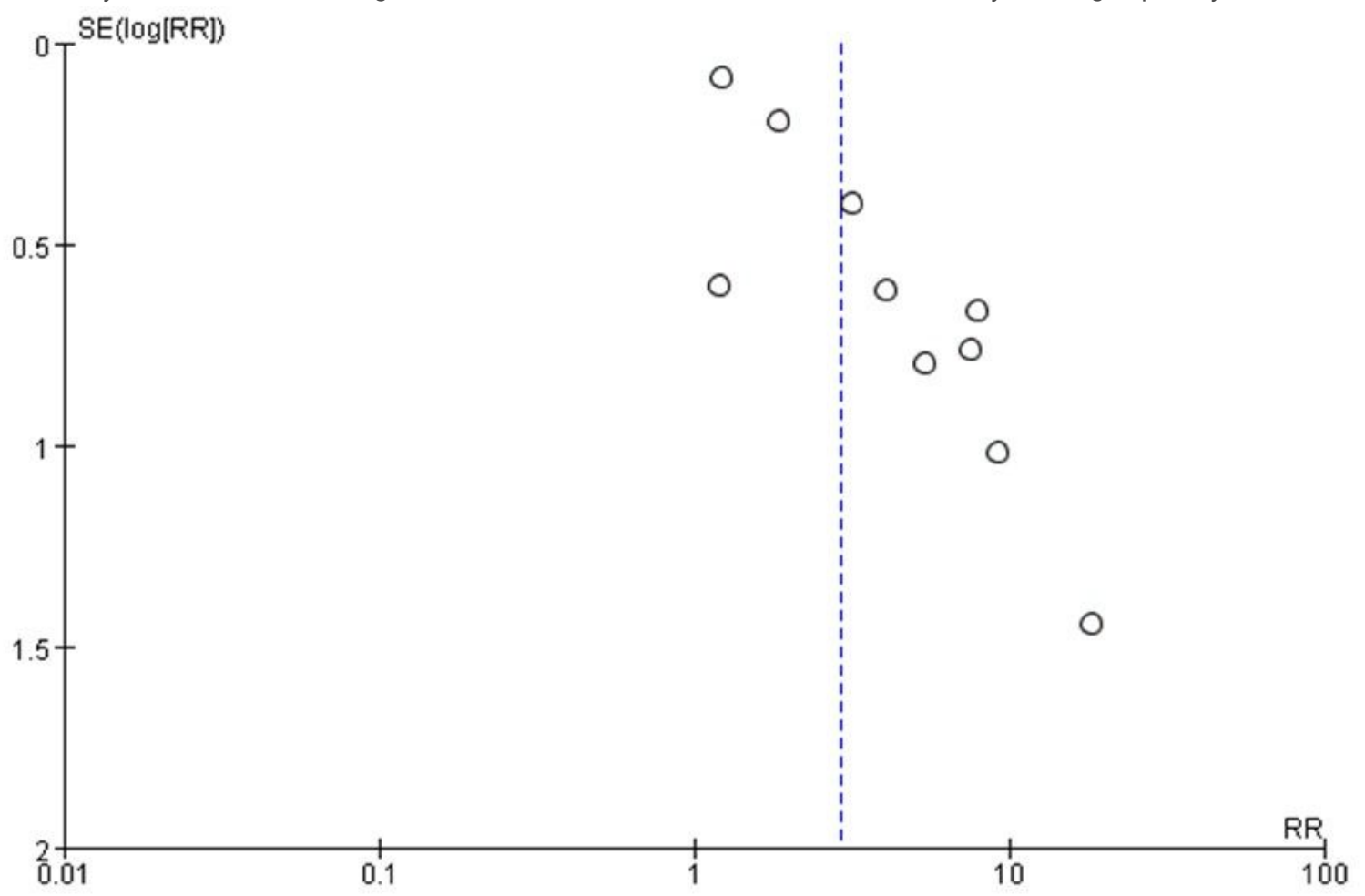

Page 10/11 
Figure 3

Funnel plot of AAV-associated-ILD and mortality.

\section{Supplementary Files}

This is a list of supplementary files associated with this preprint. Click to download.

- supplementarydata.pdf 